\title{
CORRIGENDUM
}

\section{Stage 2 of the Wellcome Trust UK-Irish bipolar affective disorder sibling-pair genome screen: evidence for linkage on chromosomes 6q16-q21, 4q12-q21, 9p21, 10p14-p12 and 18q22}

D Lambert, F Middle, ML Hamshere, R Segurado, R Raybould, A Corvin, E Green, E O’Mahony, I Nikolov, T Mulcahy, S Haque, S Bort, P Bennett, N Norton, MJ Owen, G Kirov, C Lendon, L Jones, I Jones, P Holmans, M Gill and N Craddock

Molecular Psychiatry (2006) 11, 1140-1143. doi:10.1038/sj.mp.4001917

Correction to: Molecular Psychiatry (2005) 10, 831-841. doi:10.1038/sj.mp.4001684

Following publication of the above article, the authors noticed that Figure 1 did not include a color key detailing the line colors. The key is indicated in the revised figure on pages 1141-1143. 
Chromosome 1

Chromosome 2
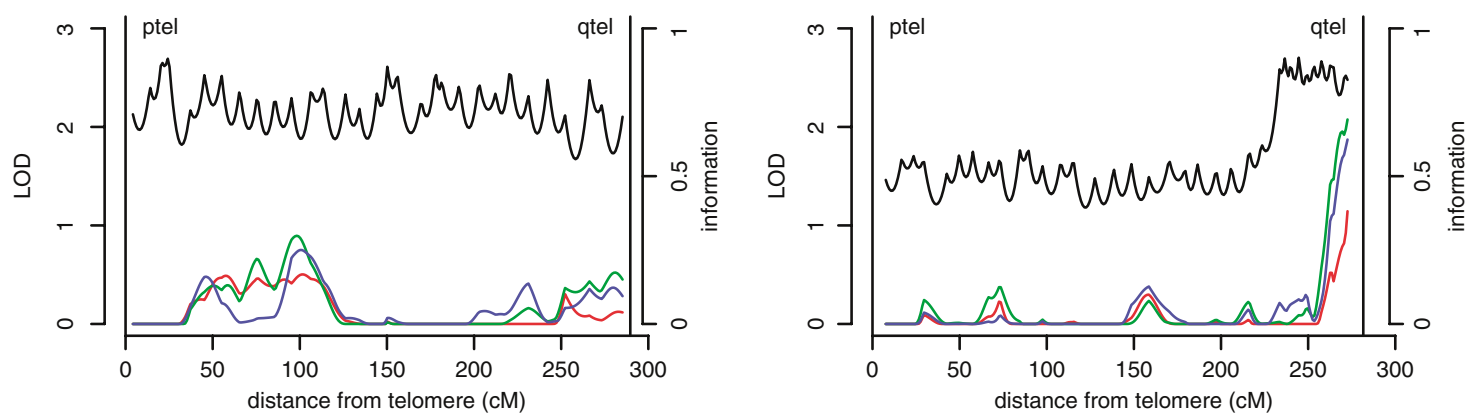

Chromosome 3

Chromosome 4
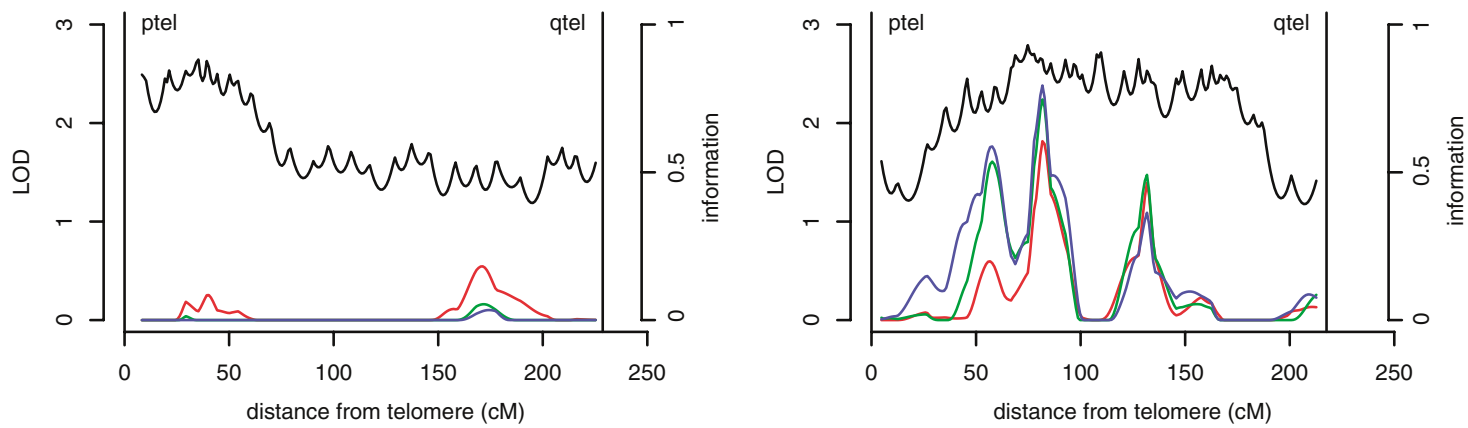

Chromosome 5

Chromosome 6
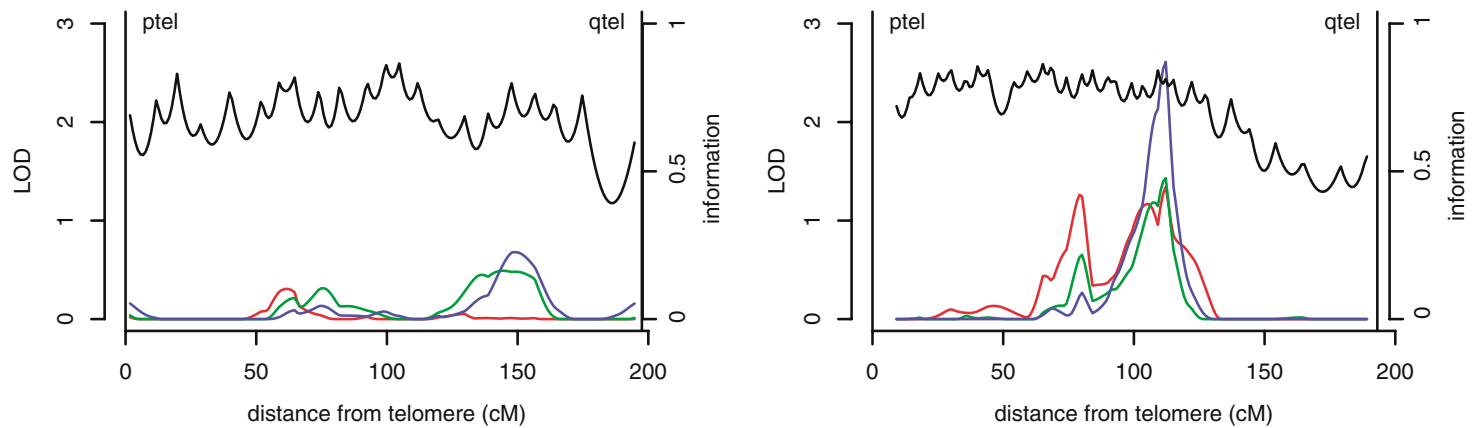

Chromosome 7

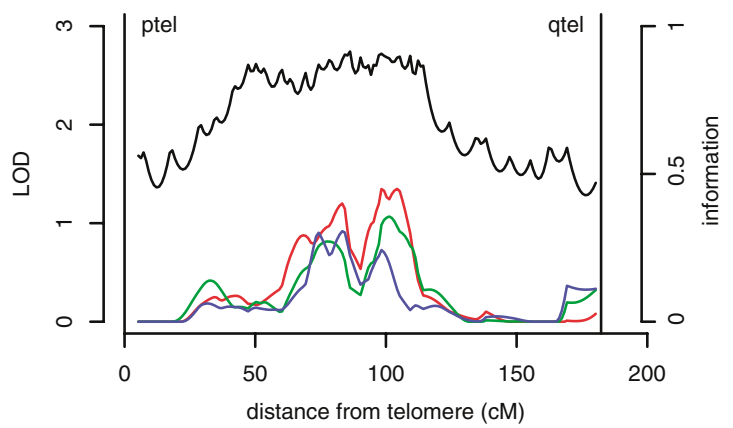

Chromosome 8

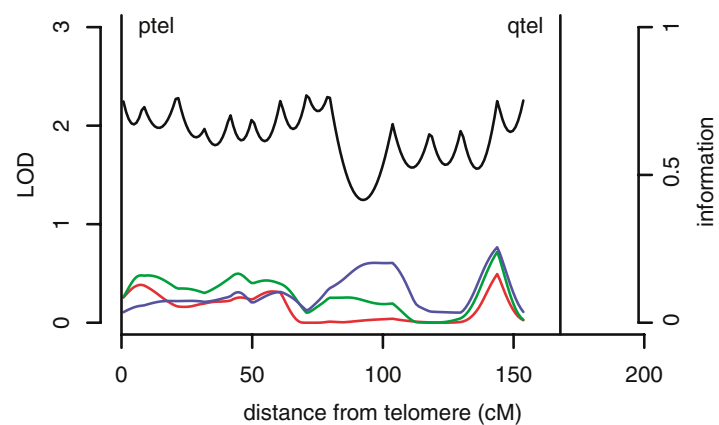

— narrow

Figure 1 Affected sibling pair maximum likelihood scores plots (MLS, primary y-axis) across all chromosomes, under each of three diagnostic models. Also shown is the marker information content (secondary $y$-axis). Distances on $x$-axis are in centiMorgan (Kosambi). (See end of figure for colour coding.) The MLS data were generated using GENEHUNTER version 2.1 for autosomes and MAPMAKER/SIBS for the X-chromosome. 
Chromosome 9

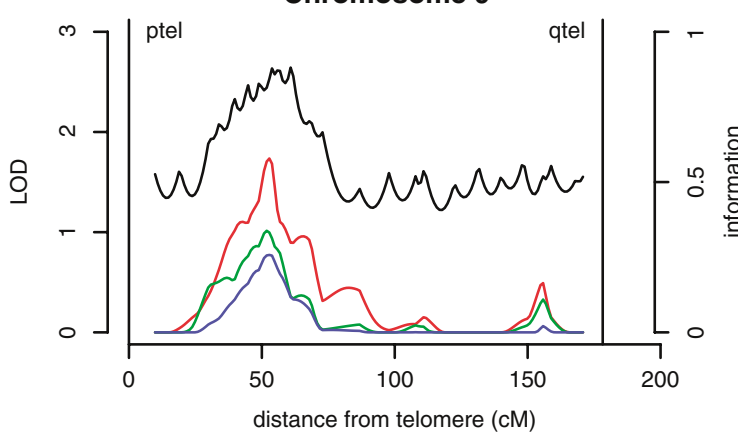

Chromosome 11

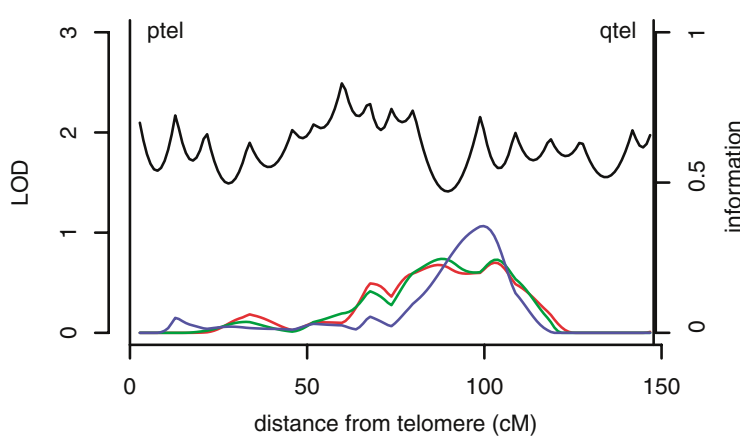

Chromosome 13

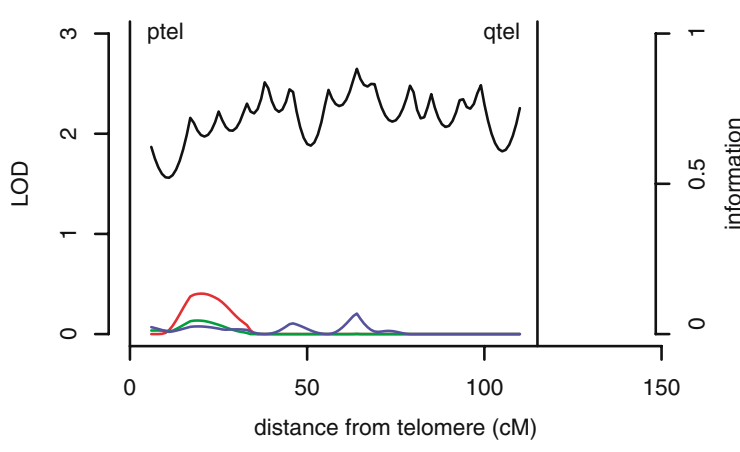

Chromosome 15

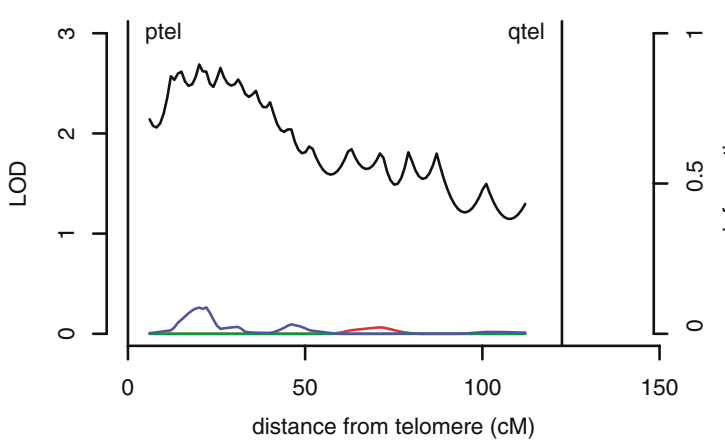

Chromosome 10

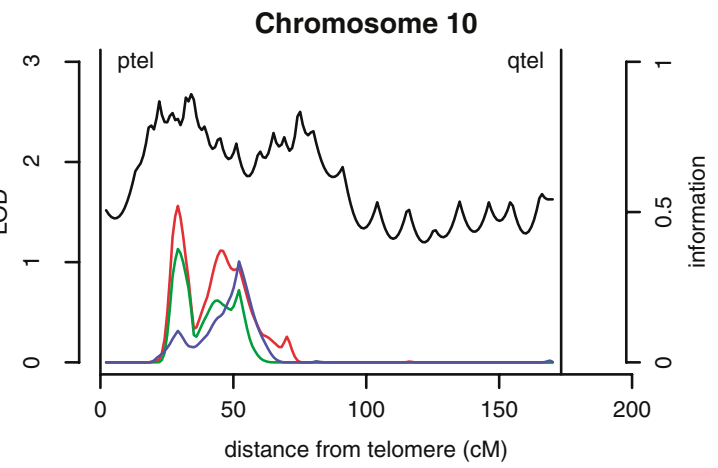

Chromosome 12

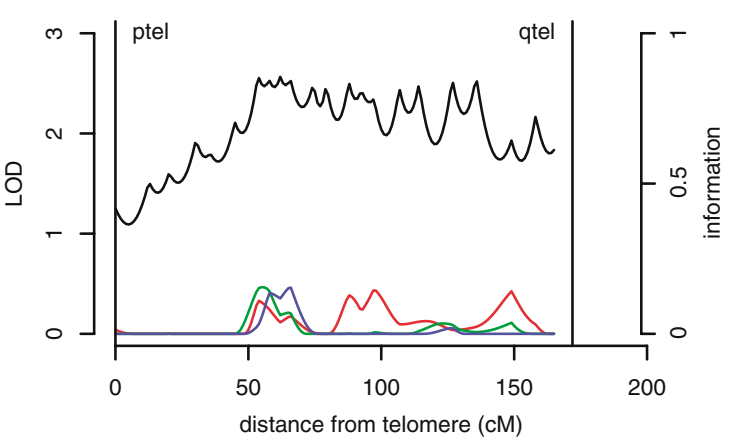

Chromosome 14

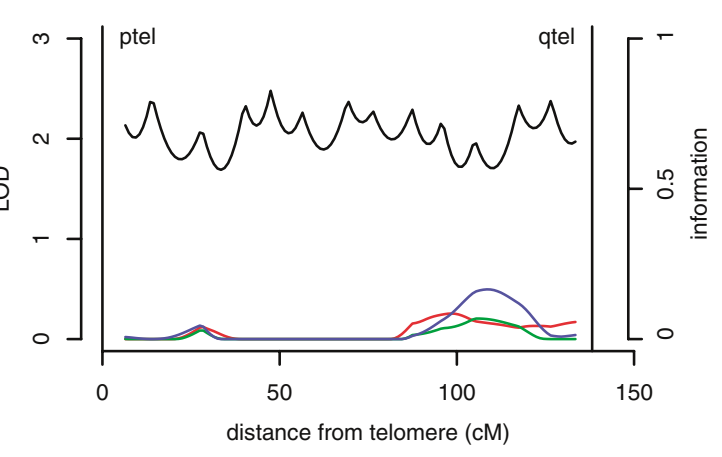

Chromosome 16

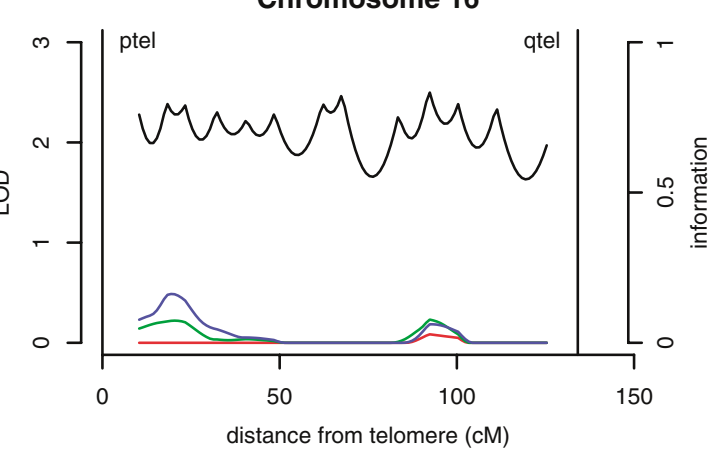

$$
\text { — narrow } \quad \text { intermediate } \longrightarrow \text { broad }
$$

Figure 1 Continued. 


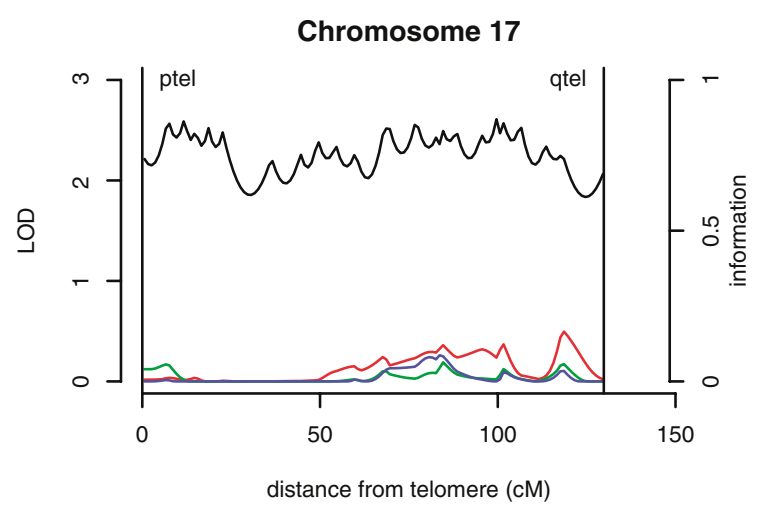

Chromosome 19

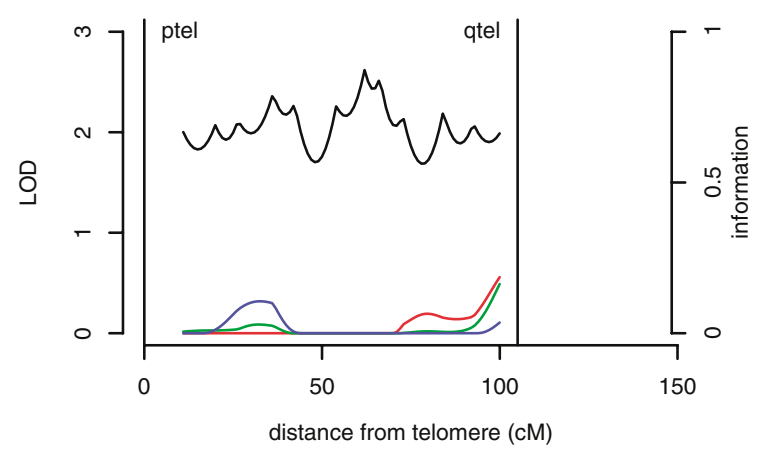

Chromosome 21

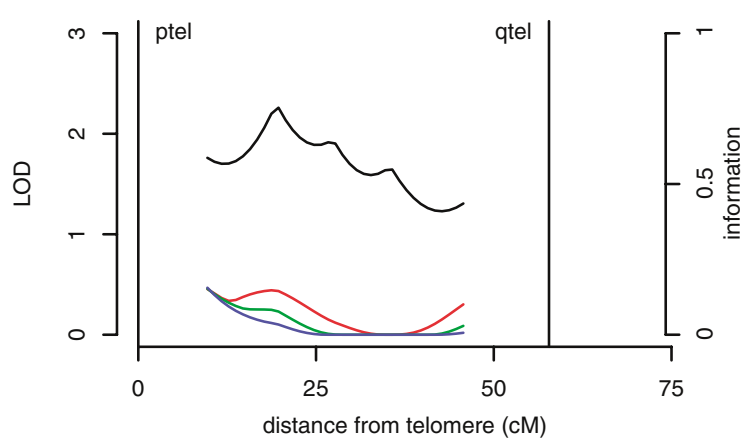

Chromosome X

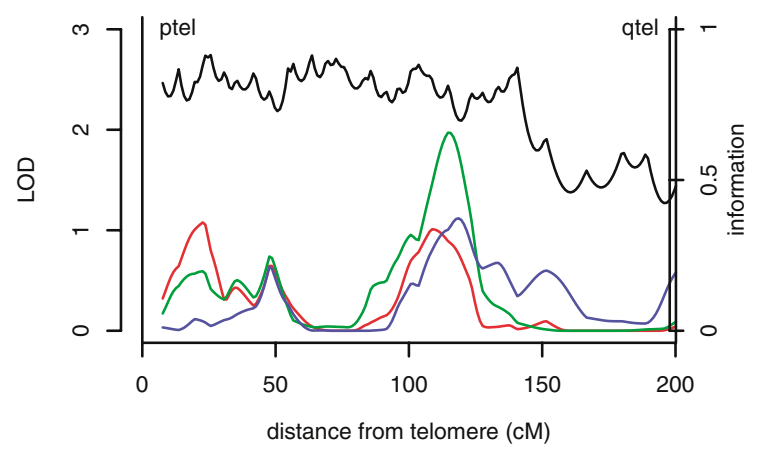

Figure 1 Continued.
Chromosome 18

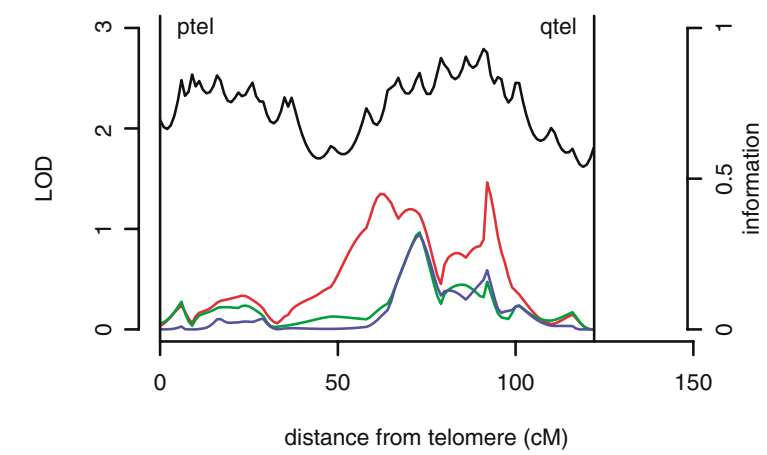

Chromosome 20

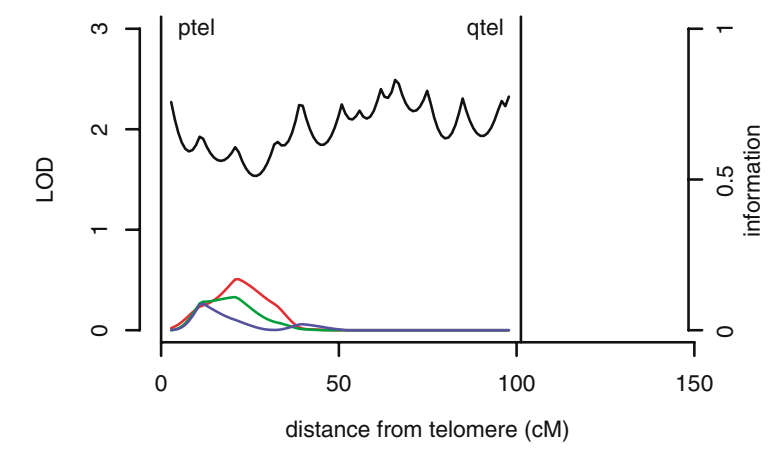

Chromosome 22

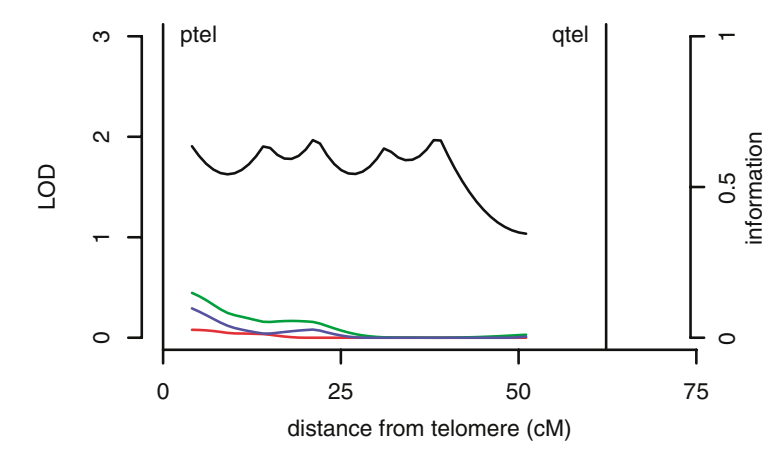

